\title{
Undergraduate labs and computers: The case for PsychExps
}

\author{
JOHN E. WILLIAMS, KENNETH O. MCGRAW, and MARK D. TEW \\ University of Mississippi, University, Mississippi
}

\begin{abstract}
PsychExps (http://www.olemiss.edu/PsychExps) is an interactive on-line psychology laboratory designed to facilitate teaching and conducting research over the Internet. With the increased use of the Web among students and the development of technology enabling the deployment of experiments via the Internet, a laboratory such as PsychExps appears to offer numerous advantages over the solely classroom-based laboratory. As part of the development of PsychExps, a survey was conducted, to assess the utility of this novel approach to the traditional computer-based psychology laboratory classes. Responses generally indicate interest in and a need for cost-effective resources such as an Internetbased laboratory. In addition, PsychExps offers several advantages over the mostly pedagogical and demonstration-based psychology sites on the Internet.
\end{abstract}

Advancements in computer technology have placed today's psychology students and professors in the unique and exciting position of being the first generation to be able to conduct truly interactive experiments via the Internet. In the past, psychology departments fortunate enough to have computer labs have based their classes around expensive hardware and software that was quickly outdated. Now, with the advent of such interactive Web sites as PsychExps (http://www.olemiss.edu/PsychExps), IPL (http://kahuna.psych.uiuc.edu/ipl/), and CogLab (http://www.psych.purdue.edu/ coglab/), students can run complex experiments from virtually all Internet-connected computers.

An immediate application of the new technology is for laboratory classes in psychology, at least for those that use experiment packages installed on local computers. Rather than depend solely on department-owned computers and software to conduct these experiments, departments can depend on student- or university-owned computers and on software provided free at Internet sites. The dollar savings in software and equipment is enhanced by valuable space savings.

In addition to financial arguments favoring the new technology, there are functional arguments. Sample sizes for research conducted in traditional department-based laboratory experiments are often limited by the size of the class itself. Since the typical lab class contains fewer than

\footnotetext{
The PsychExps project is funded by a grant from The Fund for the Improvement of Postsecondary Education (FIPSE) through the U.S. Department of Education and is supported by the University of Mississippi. This paper is based on "Psychology Experiments on the Internet: Survey of Computing and Laboratory Resources in North American Institutions" and "Psychology Experiments on the WWW: Use of Shockwave Technology," presented at the 1998 SCiP conference in Dallas. Correspondence concerning this article should be addressed to J. E. Williams, Department of Psychology, Peabody Hall, University of Mississippi, University, MS 38677 (e-mail: jewilli2@olemiss.edu).
}

20 students and the students themselves are the only research participants, lab exercises must focus on phenomena with especially large effects that can be replicated reliably in small data sets using within-subjects designs.

Internet communications remedy this problem in two ways. First, because data can be collected in parallel, the amount of data that can be generated in a fixed time period is far larger than that for experiments requiring serial subject participation. Second, data collected on a Web server can be pooled across classes far more effectively than can data collected locally. Pooling allows for potentially vast data sets, ones that are large enough to address a great number of between-subjects questions, along with theoretically interesting questions regarding effects that are reliable but small.

PsychExps is a project that uses Web technology to achieve some of the advantages inherent in Web-delivered psychology experiments. Through PsychExps, students can perform their laboratory exercises from any Netconnected computer, and departments need only provide the network-based software. The software used by these computers is delivered on demand. It is especially appealing that the software is free, multiplatform, easily modifiable, shared with users, and constantly upgraded through the communal efforts of the users.

PsychExps was developed to achieve the following objectives: (1) to permit institutions that do not presently have a computer-based undergraduate laboratory course to introduce such a course to their curriculum; (2) to allow institutions that have computer-based lab courses to upgrade their software without monetary cost; (3) to improve the data-gathering experience for students enrolled in laboratory classes; (4) to promote positive attitudes among students toward their laboratory experiences; (5) to enable students conducting independent research projects to complete a project of reasonable scope in the space of one semester; (6) to enlarge the data sets used in typical laboratory classes; (7) to permit users to customize 
the programs used to conduct laboratory experiments; (8) to provide experimental psychology programs that run on current generation computers; (9) to maintain a set of experiments that keep up with the latest multimedia programming possibilities; and (10) to allow departments that presently maintain computer stations for student laboratory use to significantly reduce their costs.

\section{ASSESSING THE NEED FOR PSYCHEXPS}

The general importance of these goals was made evident from the results of a survey conducted in Fall 1997. The survey was designed to assess the use of computer laboratories in psychology laboratory courses, the use of software designed for such labs, the interest in using an Internet-based psychology laboratory, and the ability of institutions to use such a virtual laboratory.

Using the American Psychological Association mailing list, the surveyors e-mailed the survey to 1,000 psychology departments throughout the United States and Canada. Completed surveys were returned by 236 departments. Psychology departments from 44 different states, the District of Columbia, and Canada responded to the survey. The states with the most representation were New York (7.4\%), Ohio (7.4\%), Pennsylvania (7.0\%), and California (5.3\%). Five Canadian institutions responded as well. These demographics correspond well with those listed in Anderson (1997).

The number of psychology majors at each department ranged from 2 to 2,000 (see Table 1 ). The mean was 275 , with $91 \%$ falling at 750 or below. Out of the 219 respondents indicating the use of a laboratory course, $50 \%$ offered 1 or 2 such courses, whereas the remaining $50 \%$ offered a range of 3 to 15 laboratory courses. For institutions offering B.A. and B.S. degrees, lab courses were required $90 \%$ and $93 \%$ of the time, respectively. Although $76 \%$ of the labs reported that they investigate basic phenomena in cognition and perception, $81 \%$ of the lab classes enrolled 30 or fewer students.

Seventeen departments did not offer laboratory courses; however, 15 of those departments indicated that they would offer such courses if the resources were available. The most commonly indicated reasons for not offering such courses were "lack of resources to equip such courses" $(77 \%)$ and "lack the space for such a course" $(88 \%)$. In addition,

Table 1

Additional Survey Items and Responses Regarding Demographic Information

\begin{tabular}{lc}
\multicolumn{2}{c}{ Regarding Demographic Information } \\
\hline Number of psychology majors & Response \\
Range & \\
Mean & $2-2,000$ \\
Median & 275 \\
Number of faculty in psychology & 140 \\
Range & \\
Mean & $1-52$ \\
Median & 12 \\
\hline
\end{tabular}

$59 \%$ indicated that they lacked the staff necessary for a laboratory course. Goal 1 of PsychExps addresses allowing institutions that do not currently offer laboratory courses to introduce such courses into their curriculum. PsychExps eliminates the limitations mentioned by respondents who do not currently offer laboratory courses.

Nearly all the respondents (99\%) indicated that undergraduates have access to their respective institution's computers (with Internet access) outside of the department. As well, $56 \%$ of the departments surveyed own at least one computer that meets the minimum requirements for the use of PsychExps and that undergraduates may use. As is addressed in Goal 2, departments will be able to access the latest experiments, which are free and require no setup or installation.

Goals 3 and 4 are met by allowing students to participate in interactive experiences from a variety of settings. PsychExps allows students to perform the experiments from virtually any location and provides immediate feedback on the results. Goal 5 is accomplished by allowing data collection in a relatively short amount of time. Due to the nature of the Internet, large numbers of potential participants can be reached quickly. The investigators will be able to devote the majority of their time to research design and data analysis. One concern expressed by some potential users is that the Internet connection would actually slow down the collection of data during the actual experiment. However, since the experiments are run locally on the user's computer, the Internet connection is not a factor, once the experiment has started.

The average sample size for experiments conducted in lab classes was 32 , whereas the median sample size was 25 (see Figure 1). Most sample sizes (70\%) fell at 30 or below. Goal 6 is to enlarge the data sets used in typical laboratory courses. The sample size reported by survey respondents may not be adequate to detect many theoretically interesting effects, especially when the effects are for variable interactions. Through the use of an on-line laboratory such as PsychExps, course instructors can determine the sample size used. They have the option of using solely their class data or of including data from other classes at other institutions. This quickly brings the sample size into the hundreds and, as more and more institutions sign on, the thousands.

The categories of software most commonly used to collect data in lab classes were Data Simulation, Experiment Generator, and Experimental Package software. Several respondents indicated that they used such programs as Macromedia Director and Macromedia Authorware to design their own experiments. However, many of the respondents reported that they lack the ability to customize their software adequately for their classrooms. PsychExps is based on an open model freely available to anyone who wishes to modify or add to the experiment library. All of the source code is available for download, and a template for designing experiments is provided on the PsychExps site. After opening the template in Author- 


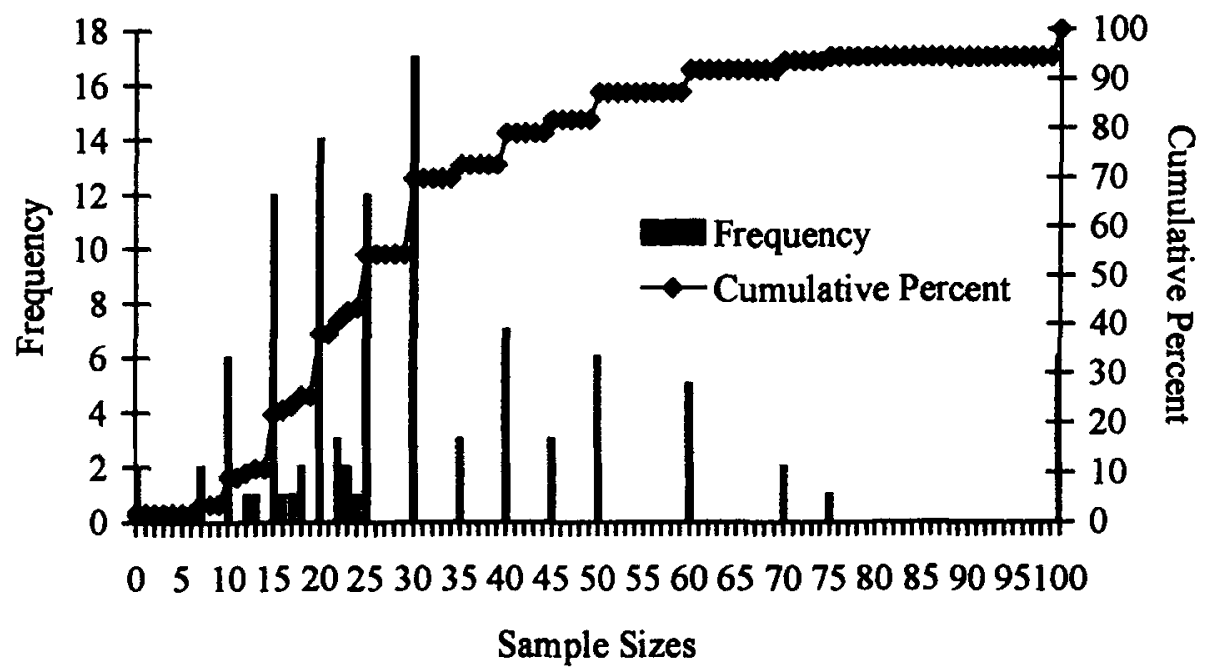

Figure 1. Report of survey respondents on sample sizes typically used in a laboratory course.

ware, the user will see annotated icons instructing them in the process of modifying or building an experiment. By meeting Goal 7, PsychExps allows instructors greater flexibility in planning, and in teaching and motivating their students. The hope is that those who choose to modify the existing programs will contribute their modifications back to the site, thereby enriching it.

Goals 8 and 9 are met by that fact that Authorware uses Macromedia's Shockwave (http://www.macromedia. $\mathrm{com} /$ ) technology. This downloadable plugin runs through the user's Web browser and brings functionality to the user's machine, even for older platforms. The users do not have to purchase the latest, up-to-date hardware in order to take advantage of multimedia and other new technology.

Funds allocated per year to maintain the laboratory software and the necessary computer hardware ranged from $\$ 0$ to $\$ 75,000$. Most respondents $(89 \%)$ were limited to $\$ 5,000$ or less, although $23 \%$ (the modal response) received no funds whatsoever. As can be seen in Figure 2, incremental increase in allocated annual funds decreases sharply after approximately $\$ 3,000$, which represents the 81 st percentile in the distribution. Goal 10 , to save money on expensive laboratory equipment, hardware, and software, is seen to be a need from the above numbers. Departments will be able to use their limited funds more effectively. PsychExps eliminates the need for expensive commercial software and the need for continuous upgrades of dedicated computer equipment.

\section{PSYCHEXPS' LIBRARY OF EXPERIMENTS AND FEATURES}

The increased use of the Internet and the development of many on-line applications leads naturally to the development of an online laboratory. PsychExps currently offers 10 experiments commonly used in laboratory courses and one template for new experiments (see Table 2).

PsychExps offers several advantages over traditional means of conducting laboratory courses and over other on-line laboratories. There are other on-line laboratories

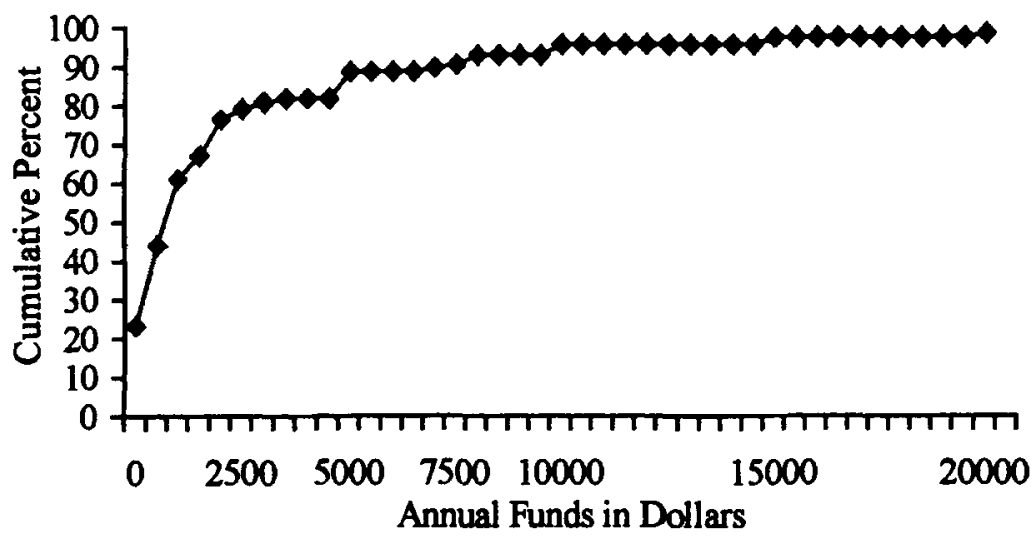

Figure 2. Annual funds allocated to laboratory software and hardware purchase and maintenance. 
Table 2

Experiments at PsychExps, Spring 1999

Müller-Lyer-Participants investigate the effects of fin orientation, fin number, and shaft length on the classic Müller-Lyer illusion by adjusting these variables.

Poggendorff-Participants determine how the magnitude of the Poggendorff illusion is affected by changes in the separation of vertical line components and changes in the length of the diagonal line components.

Reaction Time Battery-Participants investigate the effects of stimulus modality and task complexity on their ability to react. Experiments can be obtained through the combination of stimuli and decision.

Word Recognition-Participants use text entry boxes to indicate whether they can identify words that are presented tachistoscopically.

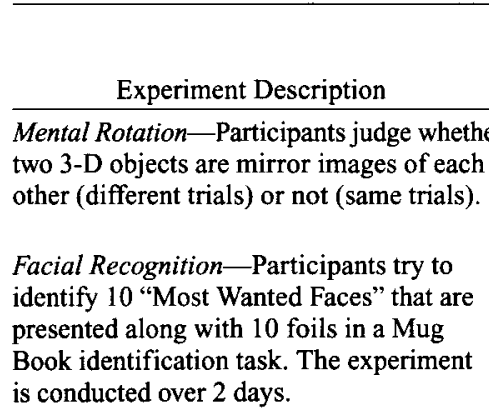

Experiments at Psychexps, Spring 1999

Subject

Variables

of Interest

Gender 1. Response time for making

Within subjects: Gender

1. Angular discrepancy between 3-D ob- Age jects, $0^{\circ}$ to $15^{\circ}$ in $45^{\circ}$ increments

2. Type of trial (same or different)

1. Between subjects: False memory condition is created by using the same foil photographs on Day 2 as on Day 1. Control condition gets fresh set of foils on Day 2.

2. Within subjects: Time (Day 1 or Day 2)

Within subjects:

1. Shaft length to fin length ratio

2. Number of fins

3. Angle of fins

Within subjects:

1. Separation of verticals (narrow, medium, and wide)

2. Length of diagonals (long and short)

Within subjects:

1. Stimulus modality (visual or auditory)

2. Type of task (Simple RT, Go/No Go RT, or Choice RT)

Within subjects:

1. Word position (right or left)

Age

Gender 1. Hits

Race 2. Misses

3. Correct rejections

4. False alarms

5. $A^{\prime}$ (a discrimination index from signal detection theory)

Difference in shaft length between user-created object and a line that the participant adjusts to be equal in length to the shaft on the user-created object

Degree of misalignment between the two diagonal line components following the user's attempt to adjust them to be collinear

Time between stimulus onset and response

1. Gender

2. Handedness

1. Exposure time for the trial on which words are identified. Exposure times start at $90 \mathrm{msec}$ and increment by $10 \mathrm{msec}$ until the word is correctly identified.

2. Accuracy of word identification in the first trial

1. Word position (left, right, or center)

2. Trial type (font color congruent with word name or noncongruent) names) that appear centrally or to the left or the right of a fixation point.

Line Motion-Participants judge whether a line drawn on the screen is drawn from right to left or left to right or whether it appears instantaneously.

Temporal Judgment-Participants judge which of two ellipses appears first along a rectangular bar that extends across the screen.

Pitch Memory-Participants listen to a sequence of six tones and indicate whether the first and last tones are the same or different.
Within subjects:

1. Position of an attentional cue that appears just before the line is drawn to the screen

2. Line speed which ranges from 0 to $100 \mathrm{msec}$.

Within subjects:

1. Time difference between the appearance of the ellipses, which ranges from 0 to $100 \mathrm{msec}$

2. Position of a prestimulus attentional cue (right, center, or left)

Within subjects:

Trial type (same or different)
1. Time between onset of the word and the user's response

2. Number correct

Percent of correct judgments

Percent correct judgments

\begin{tabular}{|c|c|}
\hline $\begin{array}{l}\text { 3. Years and } \\
\text { type of } \\
\text { musical } \\
\text { training } \\
\text { 4. Handedness }\end{array}$ & $\begin{array}{l}\text { Number correct and incorrect } \\
\text { on same and different trials }\end{array}$ \\
\hline
\end{tabular}

that offer various experiences to the user, and many of these Web sites use demonstrations or experiments similar to those found on PsychExps. Purdue University's psychology department has an on-line cognitive laboratory (http://www.psych.purdue.edu/ coglab/demos. html), which offers demonstrations written in Java, but they do not appear to collect data or allow for the manipulation of variables. The University of Illinois also has an on-line laboratory (http://kahuna.psych.uiuc.edu/ $\mathrm{ipl} /$ ) with experiments written in Java. The site offers experiments in visual and auditory processes, cognitive experiments, and others. However, although these experi- 
ments collect data, the data are not available for public use. Thus, one of the PsychExps features that continues to distinguish it from other innovative sites is the collection of data available for classroom analysis. PsychExps also provides easy-to-use Excel macros to filter and view the data sets.

Another distinguishing feature is that PsychExps will strive for group ownership of the site. The site provides the Authorware source files for download and actively encourages users to modify them and create new experiments for inclusion on the PsychExps site. PsychExps will eventually have a comprehensive library of experiments, giving appropriate credit to each experiment designer. As the site grows, the users will dictate the development and use of the site and help maintain its library. Furthermore, PsychExps is designed to facilitate a collaborative environment in which research materials and research data are seen as common property and not as the sole property of any individual user.

A final distinguishing feature is the use of Authorware. Authorware, like Java, allows the presentation of experiments via the Web. Other authoring programs, such as E-Prime, do not incorporate such technology. Although Java has great potential as a multiplatform, multibrowser interface, it is still difficult to program an experiment in Java that will run across the wide spectrum of configurations (Rahmat, 1998). Authorware, on the other hand, does not have this difficulty. The use of Authorware as a development tool allows easy manipulation and modification of experiments. Although other authoring programs and programming languages, such as Java, may be free, they often take some time to learn and may not run reliably on some machines (Miller, 1998). Without prior programming knowledge, Java would take several months to learn adequately enough to program a simple experiment. We believe that a relatively novice user could take Authorware and the template system used by PsychExps, make minor modifications to one of the experiments on PsychExps within hours, and design new experiments within weeks, if not days.

\section{CONCLUSION}

The results of our survey indicate that the academic environment would greatly benefit from the advantages that PsychExps has to offer. Although most institutions do utilize computers for their laboratory courses, the scope of these courses is limited by their departments' computer facilities. A psychology department need not have a large computer lab, the most up-to-date hardware or software, or even a large student population to conduct, analyze, or create large sets of data. Since lack of funding is the biggest obstacle between psychology departments and their use of computers to their maximum potential, PsychExps can indeed assist programs around the world in achieving optimal use of today's technology.

\section{REFERENCES}

Anderson, C. J. (ED.) (1997). Fact book of higher education. Phoenix, AZ: Oryx Press.

MilleR, M. (1998). Why Java still matters. PC Magazine [On-line], 17. Available: http://www.zdnet.com/pcmag/insites/miller_print $/ \mathrm{mm}$ 980407.htm

RAHMAT, O. (1998). The state of Java. Interactivity [On-line], 4. Available: http:/www.interactivity.com/backissues/1998/1098/rahmat9810.pdf

(Manuscript received December 15, 1998; revision accepted for publication March 25, 1999.) 